\title{
Novel functions and applications of trehalose*
}

\author{
Takanobu Higashiyama
}

\author{
Amase Institute of Hayashibara Biochemical Laboratory, Inc., 7-7 Amase Minami- \\ Machi Okayama 700-0834, Japan
}

\begin{abstract}
Trehalose is a disaccharide with important functional properties. Although these properties have been recognized for many years, trehalose was not produced on an industrial scale. Recently, a novel enzymatic system for the production of trehalose was developed, and the cost has been dramatically reduced enough to permit widespread application. In the present study, the stabilization mechanism of unsaturated fatty acids by trehalose was found. Furthermore, possible suppressive effects of trehalose on osteoporosis development emerged from our experiments. Trehalose is also expected to be used for various applications in the fields of cosmetics and pharmaceuticals.
\end{abstract}

\section{PROPERTIES OF TREHALOSE}

Trehalose is a disaccharide composed of two glucose molecules bound by an alpha, alpha-1, 1 linkage. Since the reducing end of a glucosyl residue is connected with the other, trehalose has no reducing power. Trehalose is widely distributed in nature. It is known to be one of the sources of energy in most living organisms and can be found in many organisms, including bacteria, fungi, insects, plants, and invertebrates. Mushrooms contain up to 10-25\% trehalose by dry weight. Furthermore, trehalose protects organisms against various stresses, such as dryness, freezing, and osmopressure. In the case of resurrection plants, which can live in a dry state, when the water dries up, the plants dry up too. However, they can successfully revive when placed in water. The anhydrobitic organisms are able to tolerate the lack of water owing to their ability to synthesize large quantities of trehalose, and the trehalose plays a key role in stabilizing membranes and other macromolecular assemblies under extreme environmental conditions.

The properties of trehalose are shown in Table 1. Its relative sweetness is $45 \%$ of sucrose. Trehalose has high thermostability and a wide $\mathrm{pH}$-stability range. Therefore, it is one of the most stable saccharides. When $4 \%$ trehalose solutions with 3.5 to $10 \mathrm{pH}$ were heated at $100{ }^{\circ} \mathrm{C}$ for $24 \mathrm{~h}$, no degradation of trehalose was observed in any case. Because of nonreducing sugar, this saccharide does not show Maillard reaction with amino compounds such as amino acids or proteins. Its particular physical features make it an extremely attractive substance for industrial applications. Furthermore, this saccharide shows good sweetness like sucrose, and in the food industry, this saccharide is used as a sweetener.

*Pure Appl. Chem. Vol. 74, No. 7, 2002. A special topic issue on the science of sweeteners. 
Table 1 Properties of trehalose.

\begin{tabular}{lll}
\hline Melting point & $\begin{array}{l}\text { dihydrate } \\
\text { anhydride } \\
\text { dihydrate } \\
\text { Heat of fusion }\end{array}$ & $\begin{array}{l}97.0^{\circ} \mathrm{C} \\
\text { anhydride }\end{array}$ \\
Solubility & $68.9 \mathrm{~g} / 100 \mathrm{~g} \mathrm{H}_{2} \mathrm{O}$ at $20{ }^{\circ} \mathrm{C}$ \\
Relative sweetness & $45 \%$ of sucrose & $57.8 \mathrm{~kJ} \mathrm{~mol}^{-1}$ \\
Digestibility & $53.4 \mathrm{~kJ} \mathrm{~mol}^{-1}$ \\
pH stability of solution & $>99 \%\left(\mathrm{pH} 3.5-10\right.$, at $100{ }^{\circ} \mathrm{C}$ for $\left.24 \mathrm{~h}\right)$ & \\
Heat stability of solution & $>99 \%\left(\right.$ at $120{ }^{\circ} \mathrm{C}$ for $\left.90 \mathrm{~min}\right)$ & \\
\hline
\end{tabular}

\section{PRODUCTION OF TREHALOSE}

Although its usefulness was recognized, trehalose was not produced on an industrial scale until 1994. The conventional method for production, for example, extraction from yeast, had too low a yield and too high a cost to be used. In order to implement industrial production of trehalose, we have researched new enzyme systems and have succeeded in isolating a novel enzyme system from a bacterial strain belonging to the genus Arthrobacter sp. Q36 that was obtained from soil [1]. The system has been found to consist of two novel enzymes: malto-oligosyltrehalose synthase (or MTSase) and malto-oligosyltrehalose trehalohydorolase (or MTHase). In the first step, MTSase catalyzes the intramolecular transglycosylation of glucose residue at the reducing end of malto-oligosaccharide from $\alpha-1,4$ bond to $\alpha-1,1$ bond, then malto-oligosyltrehalose is produced. This contains trehalose residue at the end of the saccharide chain. Next, trehalose is liberated from malto-oligosyltrehalose by MTHase. This pathway needs no high-energy sugar derivative such as sugar phosphate or sugar nucleotide. And those enzymes can repeatedly act on $\alpha-1,4$ glucan to produce trehalose up to $80 \%$ yield (Fig. 1).

The trehalose production scheme that has been developed in our company is shown in Fig. 2. Starch is liquefied by a thermostable $\alpha$-amylase and is debranched by isoamylase. The formed amylose is converted into trehalose by this system. The system involves two novel enzymes, MTSase and MTHase, which act on amylose or starch to produce trehalose efficiently. By this process, we have succeeded in reducing the production cost of trehalose to approximately one hundredth of its initial cost. The cost was reduced enough to permit a widespread application to many foods such as sweeteners and stabilizers. In addition, trehalose is also expected to be used for various applications in the fields of medicine and cosmetics.

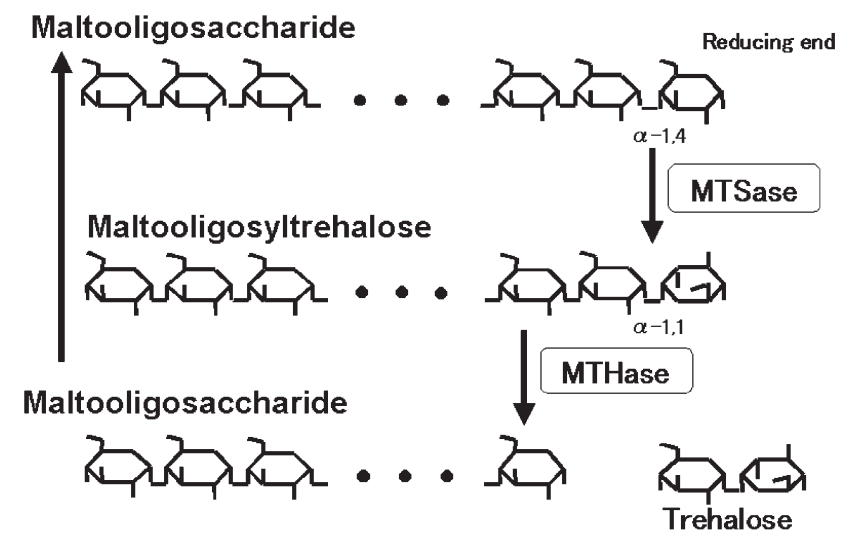

Fig. 1 Scheme of trehalose production by MTSase and MTHase. 


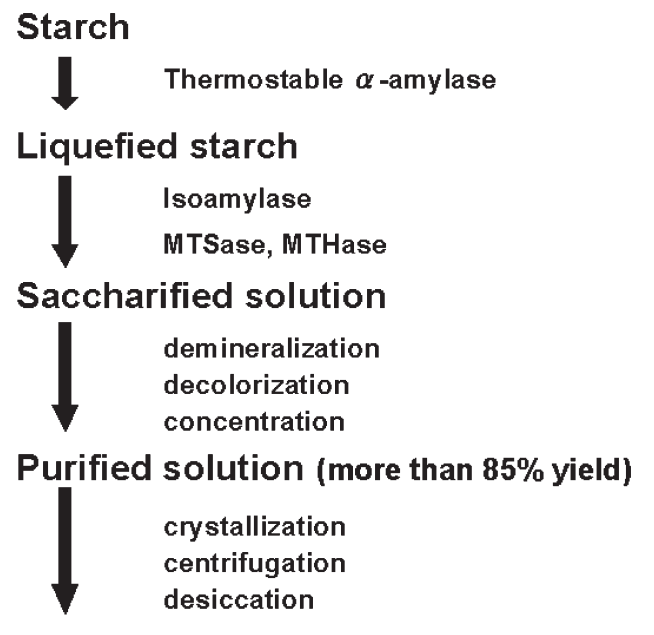

Trehalose (dihydrate crystals $\mathbf{9 8 \%}$ purity)

Fig. 2 Production of trehalose from starch.

\section{EFFECT OF TREHALOSE ON THE DEGRADATION OF FATTY ACID}

In the present study, oligosaccharide's application as a sweetener has been investigated together with its functions. Carbohydrates, proteins, and lipids are three important sources of nourishment. Trehalose has good stabilizing functions, namely, preventing starch retrogradation, protein denaturation, and lipid degradation. For example, trehalose shows poor reactivity against amino compounds in food, because it has high thermostability, wide $\mathrm{pH}$-stability range, and no reducing power. It also masks unpleasant tastes and odors in food. So it is superb for the maintenance of food quality.

We recently found that trehalose is capable of suppressing degradation of fatty acid. Lipid oxidation by heating and exposure to air is the cause of a peculiar odor called degradation odor. Furthermore, it is known that oxidation products of lipids have a bad influence on human health and aging. One of the three fatty acids $(100 \mathrm{mg})$ that have different amounts of double bond was mixed with $1 \mathrm{ml}$ of a $5 \%$ solution of 6 types of disaccharides or without adding a sugar solution (control). After the saccharides and fatty acids were mixed in a vial, the mixture was heated to boil for $1 \mathrm{~h}$. The volatile aldehydes in the head-space gas (HSG) of the vial were measured using gas chromatography. The effect of saccharides on the formation of volatile aldehydes from $\alpha$-linolenic acid is shown in Table 2, as an example. The generation of almost all volatile aldehydes, propanal, butanal, and hexa-

Table 2 Effect of saccharides on the formation of volatile aldehydes from $\alpha$-linolenic acid.

\begin{tabular}{lccc}
\hline Saccharide & \multicolumn{2}{c}{ Volatile aldehydes $(\mu \mathrm{g} / \mathrm{ml}-\mathrm{HSG})$} \\
\cline { 2 - 4 } & Propanal & Butanal & Hexanal \\
\hline Non add. (control) & 10.00 & 0.23 & 0.11 \\
Trehalose & 1.27 & 0.07 & 0.02 \\
Sucrose & 9.51 & 0.23 & 0.09 \\
Maltose & 9.40 & 0.19 & 0.10 \\
Neotrehalose & 8.60 & 0.19 & 0.08 \\
Maltitol & 2.44 & 0.08 & 0.02 \\
Sorbitol & 8.00 & 0.19 & 0.09 \\
\hline
\end{tabular}


nal was remarkably suppressed to $10-30 \%$ of the control by the addition of trehalose, but was not influenced by the addition of sucrose or sorbitol. In the case of the effect of saccharides on the formation of 2,4-decadienal from linoleic acid by boiling, trehalose's suppressive effect on the generation of volatile aldehydes was also found (Table 3). In short, the above-mentioned results show that masking of odors in food is caused by the suppressive action of trehalose.

It is supposed that trehalose has a suppressive effect on the autooxidation of unsaturated fatty acids. As shown in Fig. 3, the formation of hydroperoxide (HPOD), which is the initial reaction product of linoleic acid oxidation, was suppressed by trehalose. So it is considered that trehalose might directly interact with linoleic acid. As a result, the formation of hydroperoxide is suppressed. In order to prove this mechanism experimentally, we measured relaxation time of linoleic acid, trehalose, and their mixture by NMR analysis. The observed relaxation time provides atomic movement. The relaxation time decreased with limiting atomic movement by some interaction. The relaxation time of 9,10 , 12, 13 positions on olefin of linoleic acid decreased by the addition of trehalose (Fig. 4). Therefore, it is supposed that trehalose might interact with olefin residue in linoleic acid. The relaxation time of trehalose only and trehalose with linoleic acid by ${ }^{13} \mathrm{C}-\mathrm{NMR}$ analysis is shown in Fig. 5. The relaxation time of 3, 6 positions of trehalose decreased, owing to the interaction with linoleic acid. Furthermore, when the ratio of linoleic acid to trehalose was over 1:2, the relaxation time of linoleic acid leveled off (Fig. 6). This result shows that 1 molecular trehalose interacts with 1 olefin. Therefore, we can say that linoleic acid was stabilized. These results suggest the existence of an interaction between 3, 6 positions of two trehalose molecules and 9, 10,12,13 positions of the linoleic acid molecule. It is considered that the interaction with trehalose may stabilize the olefin residue of linoleic acid against oxidation.

Table 3 Effect of saccharides on the formation of 2,4-decadienal from linoleic acid.

\begin{tabular}{lc}
\hline Saccharide & 2, 4-decadienal \\
\hline Non add. (control) & 16.70 \\
Trehalose & 1.93 \\
Sucrose & 16.00 \\
Maltose & 12.40 \\
Neotrehalose & 16.30 \\
Maltitol & 2.46 \\
Sorbitol & 15.20 \\
\hline
\end{tabular}

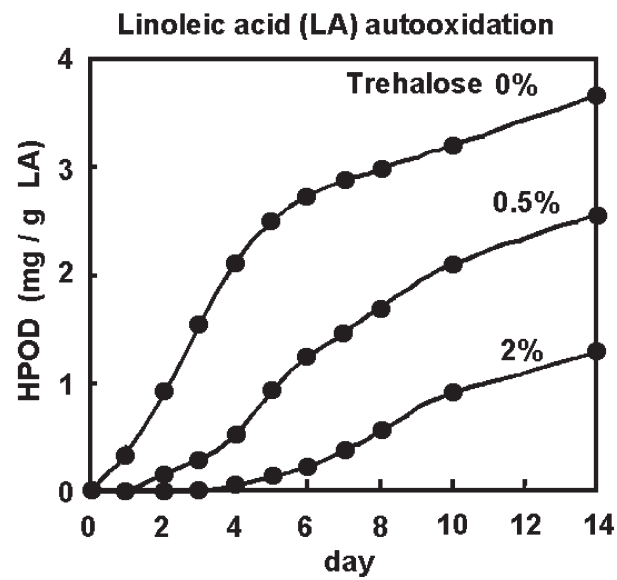

Fig. 3 Effect of trehalose on the autooxidation of unsaturated fatty acids. 


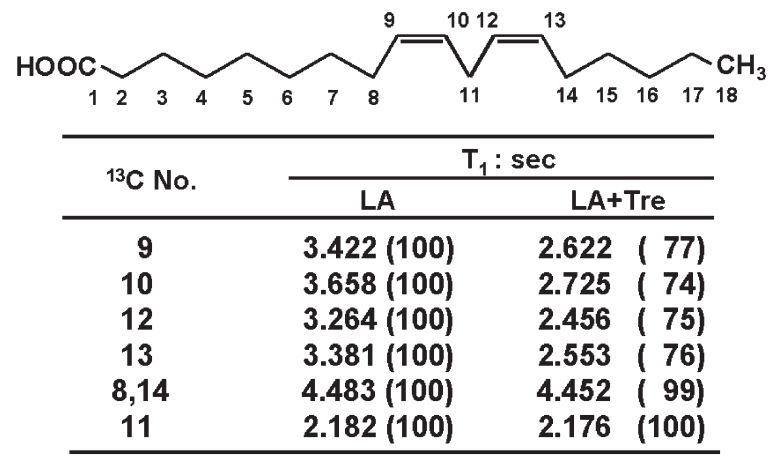

LA: Linoleic acid, Tre: Trehalose

Fig. 4 Relaxation time $\left(\mathrm{T}_{1}\right)$ of linoleic acid in ${ }^{13} \mathrm{C}-\mathrm{NMR}$ analysis.

\begin{tabular}{ccc} 
& \multicolumn{2}{c}{$\mathrm{T}_{1}: \mathrm{msec}$} \\
\hline${ }^{13} \mathrm{C}$ No. & $\mathrm{Tre}$ & $\mathrm{Tre}+\mathrm{LA}$ \\
\hline 1 & $354.79(100)$ & $347.13(98)$ \\
2 & $322.76(100)$ & $307.91(95)$ \\
3 & $368.95(100)$ & $332.32(90)$ \\
4 & $360.74(100)$ & $360.62(100)$ \\
5 & $352.13(100)$ & $333.83(95)$ \\
6 & $219.14(100)$ & $180.78(82)$ \\
\hline
\end{tabular}

Fig. 5 Relaxation time $\left(\mathrm{T}_{1}\right)$ of trehalose in ${ }^{13} \mathrm{C}-\mathrm{NMR}$ analysis.

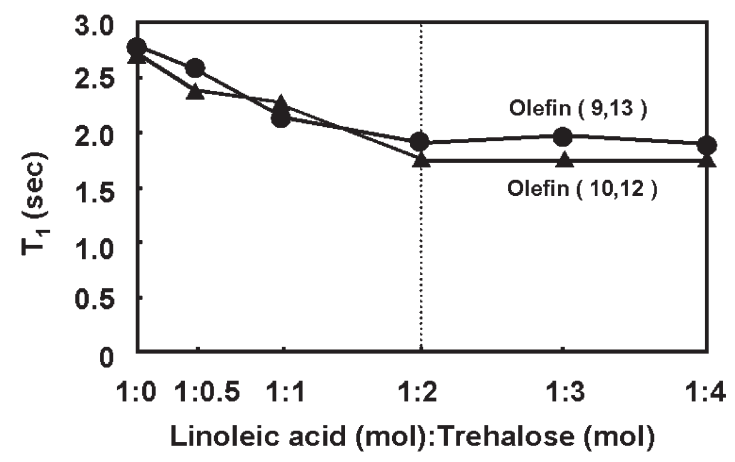

Fig. 6 Effect of trehalose on the relaxation time of linoleic acid $\left({ }^{1} \mathrm{H}-\mathrm{NMR}\right.$ analysis).

\section{SUPPRESSIVE EFFECT OF TREHALOSE ON HUMAN BODY ODOR}

In this application, we examined the suppressive effect of trehalose on human body odor. The typical odor of a senior layer (odor from seniors) increases with age, especially 55 years or older. This odor contains unsaturated aldehydes such as 2-nonenal and 2-octenal. These aldehydes are produced by the degradation of unsaturated fatty acid (palmitoleic acid) in aged people's skin. The subjects (55 years or

(C) 2002 IUPAC, Pure and Applied Chemistry 74, 1263-1269 
older) were selected from our company. After a shower, their body was sprayed with a $2 \%$ trehalose solution. They put on new underwear after the spray. Twenty hours later, the unsaturated aldehydes were sampled from the used underwear shown in this system using DNPH-column. The trapped aldehydes were eluted from this column and were analyzed by gas chromatography. The results showed a decrease of about $70 \%$ in odor from seniors due to the action of trehalose (Fig. 7). This result indicates that trehalose has a suppressive effect on the formation of the odor released by the seniors' bodies. The same results came out with the oxidation of fatty acid. Therefore, the application of trehalose for cosmetic fields is expected.

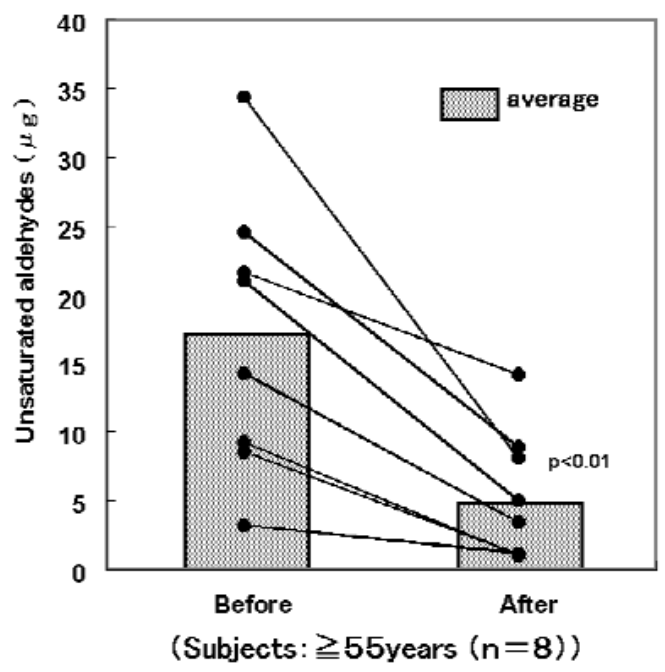

Fig. 7 Suppressive effect of trehalose on the formation of human body odor.

\section{PHYSIOLOGICAL FUNCTION OF TREHALOSE}

In recent research, the physiological function of trehalose on bone resorption in ovariectomized mice (OVX) as a model of osteoporosis was examined. As shown in Fig. 8, the trabeculae of OVX mice was significantly decreased, compared with control. In contrast, OVX mice having trehalose did not show any sign of decrease of the trabeculae. These results suggest that trehalose might have a kind of suppressive effect on the development of osteoporosis [2]. These results further imply that the daily ingestion of trehalose-containing foods could be useful both for bone metabolism and prevention of osteoporosis.

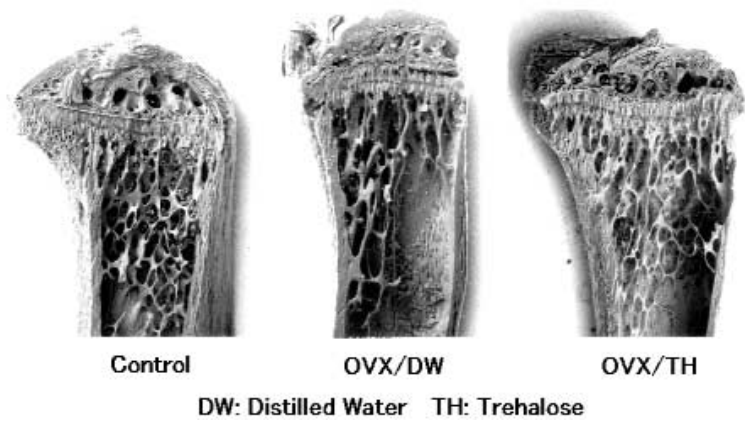

Fig. 8 Trehalose inhibits bone resorption in ovariectomized mice. 
Furthermore, it has been shown that trehalose could protect corneal epithelial cells in culture from death by desiccation and suppress tissue denaturalization [3,4]. Trehalose has been considered as a potential new drop for dry eyes syndrome and for effective preservation of organs.

\section{REFERENCES}

1. K. Maruta, T. Nakada, M. Kubota, H. Chaen, T. Sugimoto, M. Kurimoto, Y. Tsjisaka. Biosci. Biotech. Biochem. 59, 1829-1834 (1995).

2. Y. Nishizaki, C. Yoshizane, Y. Toshimori, N. Arai, S. Akamatsu, T. Hanaya, S. Arai, M. Ikeda, M. Kurimoto. Nutrition Res. 20, 653-664 (2000).

3. T. Matuo. Br. J. Ophthalmol. 85, 610-612 (2001).

4. T. Hirata, T. Fukuse, C. J. Liu, K. Muro, H. Yokomise, K. Yagi, K. Inui, S. Hitomi, H. Wada. Surgery 115, 102-107 (1994). 